\title{
Arte y violencia: la representación del daño como forma de reconstrucción de una nueva sociedad
}

\author{
Art and violence: the representation of damage \\ as a way of rebuilding a new society
}

\section{Resumen}

El desarrollo del siguiente artículo busca explorar la relación entre arte y violencia, identificando el arte como un hecho de la vida del hombre, en el cual se puede construir un mejoramiento de las condiciones de vida en las que se desenvuelve. El arte permite la representación de la interioridad subjetiva, que se revela a los otros por medio de diferentes experiencias estéticas. Se hará énfasis en la propuesta de construir una sensibilidad que permita reconocer el daño ocasionado por la violencia a las víctimas como forma de restitución de su derecho a la justicia en un ambiente de noviolencia. Inicialmente, se desarrollará la idea de una nueva forma de sociedad en la que prime el beneficio de sus miembros con un medio ambiente propicio para el desarrollo de la libertad. Seguidamente, se presentará la idea de la experiencia sublime como posibilidad de realización de la expresión subjetiva. Por último, se hará un acercamiento a obras de arte colombianas inscritas en el marco del concepto de violencia resaltando la importancia que estas tienen en el marco de la construcción de un nuevo ambiente social de cultura de noviolencia.

Palabras clave: sensibilidad, ethos estético, violencia, nueva sociedad.

\begin{abstract}
This article wants to explore the relationship between art and violence, identifying art as a fact of human life, which can be used to build the improvement of living conditions. Art allows the representation of subjective introspection, which is revealed to others through different aesthetic experiences. This article proposes to build a sensitivity that allows recognizing the damage caused by violence to victims as a form of restoring their right to justice in an atmosphere of nonviolence. Initially, this paper develops the idea of a new form of society in which the benefit of its members is a top priority with an environment conducive to the development of freedom. Additionally, this article presents the idea of the sublime experience as the possibility of realization of the subjective expression. Finally, this document takes a closer look at Colombian art works that are part of the concept of violence, highlighting the importance they play in the context of building a new social culture of nonviolence.
\end{abstract}

Keywords: sensibility, ethics-aesthetic, violence, new society.

Recibido el 23 de mayo de 2011 y aprobado el 25 de mayo de 2011

1 Giovanny Moisés Pinzón Perilla profesional en filosofía, licenciado en filosofía, especialista en bioética, candidato a magister en filosofía. Se ha desempeñado como docente de la licenciatura en filosofía, del programa de trabajo social y como Director de la especialización en ética de Uniminuto. Actualmente me desempeño como docente de la UPN. Correo electrónico: gmpperilla@gmail.com 


\section{Estética y libertad}

$\dot{c}$

Es posible pensar en una sociedad en la que el desarrollo de la libertad de sus ciudadanos sea verdaderamente real? Seguramente, si nos referimos a diversas sociedades en la que sus estados de derecho han legislado en relación a su concepto de libertad, eso sea viable. Pero de hecho, al confrontarnos directamente con algún contexto determinado, esto no sea tan real, y más cuando nos referimos a sociedades qua han vivido, viven y posiblemente vivirán en la violencia. De ahí que parezca sugerente el pensar en una nueva forma de sociedad en la que prime el beneficio de sus miembros con un medio ambiente propicio para el desarrollo de la libertad.

Esta idea de una nueva forma de sociedad, suscrita por Marcuse (1969) en un Ensayo sobre la liberación, constituye un referente para pensar en una sociedad en la que el arte o mejor dicho, la obra de arte, sea la misma sociedad que se reconstruye. Aquí el marco de inspiración es una nueva sensibilidad cuyo presupuesto de crear una sociedad libre en la que sus logros permitan dirimir la violencia.

La nueva sensibilidad, que expresa la afirmación de los instintos de vida sobre la agresividad y la culpa, nutriría en una escala social, la vital urgencia de la abolición de la injusticia y la miseria, y configuraría la ulterior evolución del nivel de la vida (Marcuse, 1969, p. 30).

La violencia y la explotación que se ejerce desde las más grandes esferas, desde la construcción de las políticas, que sólo apuntan al desarrollo económico, sin tener presente el beneficio de los miembros de la sociedad; las violencias organizadas por la pugna entre los intereses de los diferentes miembros de una sociedad, sólo terminan agobiando y maltratando el derecho a la libertad de los miembros de la sociedad. Esto nos exige el planteamiento de una lucha contra estas formas de violencia que se han mantenido en la vida de las sociedades.

La lucha que se ejerce en contra de estas formas tradicionales de violencia, es una que se encamina a lograr modos y formas de vida esencialmente nuevos, que se sintetiza en la afirmación por construir una sociedad nueva en la que la abolición de toda forma de violencia desemboquen en un mundo donde las sensibilidades se manifiesten y construyan una nueva sociedad.

[...] negación total del sistema establecido, de su moralidad y de su cultura; afirmación del derecho a construir una sociedad en la que la abolición de la violencia y el agobio desemboque en un mundo donde lo sensual, lo lúdico, lo sereno y lo bello lleguen a ser formas de existencia y, por tanto, la forma de una sociedad misma" (Marcuse, 1969, p. 32).

La expresión de las sensibilidades por parte de quienes se realizan en el mundo en el que viven, genera la posibilidad de la realización de la libertad y de la construcción de un nuevo estilo de vida, que se convierte en una obra colectiva 
en tanto que, se construye una vida hecha a partir del beneficio de las personas que habitan el mundo que han imaginado.

La imaginación en la apuesta de la nueva sensibilidad $^{2}$ política, permite desenvolver la realización de una sociedad que se emancipa de las tradicionales formas de enajenación y alienación de las clases o de los grupos imperantes, que han reprimido por sus diversas normatividades el desarrollo de la libertad de los particulares.

La dimensión estética puede servirnos como una especie de calibrador para una sociedad libre. Donde las relaciones humanas no están mediadas por el mercado, o están a la base de la explotación competitiva, sino que exige una sensibilidad receptiva de nuevas formas y modos de la imaginación estética.

La moralidad que se expresa en esta nueva forma de sociedad está situada en una experiencia orgánica: la de ser físicamente incapaz de tolerar cualquier represión que no sea la requerida para la protección y el mejoramiento de la vida. Siendo la fuerza orientadora de la calidad de vida el hecho de pensar una sociedad estéticamente posible.

El universo estético se constituye en el mundo en el que viven, en el que las necesidades y realidades para el desarrollo de la libertad se hace realmente efectivo, configura la necesidad de crear colectivamente un medio ambiente cuya Forma expresiva sea el de la libertad.

En la reconstrucción de la sociedad para el logro de esa meta, la realidad asumiría un conjunto de Forma expresiva de la nueva meta. La cualidad esencialmente estética de esa forma haría de ella una obra de arte, pero en la medida en que la forma ha de aparecer en el curso del proceso social de producción, el arte habría cambiado su sitio y funciones tradicionales en la sociedad: se habría convertido en una fuerza productiva en la transformación a un tiempo material y cultural (Marcuse, 1969, p. 34).

La idea de que la obra se puede constituir como un espacio de realización de la libertad, y de la generación de una cultura nueva, de un medio ambiente nuevo, como el de una sociedad no violenta, es parte de la perspectiva de que lo estético, en su implicación social, tiene un papel más trascendente que se evidencia con la capacidad persuasiva ante las personas para generar una nueva sensibilidad ante los hechos o realidades que acontecen en el diario vivir de una sociedad.

Se parte de la idea de que la obra de arte posee una capacidad persuasiva, en cuanto a la sensibilidad de las personas, en especial, cuando se trata de construir una sociedad con una cultura de noviolencia. La experiencia ante una obra de arte que permite, en un contexto de violencia, no perder la memoria histórica, y el hecho de relatar por medio del arte la injusticia vivida en otros tiempos, y que de manera más relevante, la obra de arte que en su contenido manifiesta o pronuncia un hecho de violencia, posibilita la manifestación de sentimientos que afectan la vida ética y política de la sociedad.

Se genera cierta percepción de quienes reciben el impacto de la obra para identificar lo violento y las injusticias vividas, aflorando una sensación de dolor y de desencanto, que se puede verter en consolidar una formación que favorezca una cultura de noviolencia en el marco de la experiencia sublime que se halla en el hecho del anonadamiento expreso en cambio de actitudes $y$ de conciencia en el mundo en el que se vive tal violencia.

2 Marcuse (1969) presenta una propuesta política que incluye una nueva forma de construir un medio ambiente propicio para la superación de la violencia y el agobio de la sociedad capitalista. Esta nueva forma se construye a partir de un ethos estético, que involucra nuevas formas de construir socialmente la realidad desde la libertad, a partir de una concepción de arte des-sublimado de la tradición histórica de opresión y negación. Para alcanzar tal fin, el autor construye la idea de una nueva sensibilidad a partir de la base de un cambio en la concepción política de las sociedades contemporáneas y postula la idea estética como forma posible de una sociedad libre.

Polisemia No. 12, 84 - 90, Arte y violencia: la representación del daño como forma de reconstrucción [...]. Bogotá, ISSN: 1900-4648. Julio - Diciembre de 2011 
La sensibilidad que se descubre ante el encuentro con la obra de arte que contiene violencia permite vislumbrar una confrontación con la realidad, que puede persuadir a las personas y llevarlas a tomar una nueva actitud ética, de transformación de sus entornos y de sus realidades más próximas. La conciencia de una experiencia transformadora de los hombres y mujeres que desean una sociedad más justa, más bella. Es una idea que representa la relación entre lo bello y lo político, que permite concebir una idea de sociedad más integral en la que se pueda realizar efectivamente la libertad de las personas, desde la persuasión de la ética, política y, porque no, estética.

\section{Lo sublime y la violencia}

El planteamiento de una nueva sociedad, cuyo medio ambiente favorezca el desarrollo de la libertad, que hace énfasis en una sociedad que en su forma estética contempla la continua manifestación de las sensibilidades de quienes viven en ella. Es conveniente desarrollar en el marco de la presentación de este trabajo, cómo la idea de la experiencia sublime se presenta como posibilidad de realización de la expresión subjetiva.

La fuerza persuasiva de las normatividades del Estado está asignada a la ley, a la norma, lo cual puede generar una aversión por parte de las personas a la imposición de la ley, por concebirla por fuera de sí, como aislada a la realización de su libertad. Es precisamente en este marco donde la fuerza persuasiva de la política se ve limitada, por carecer de una fuerza que toque las realidades de las personas, como si las cuestiones políticas ya estuviesen acabadas.

Esto se traduce en la poca realización que tiene lo legislativo en el contexto social, en especial cuando se hace referencia a situaciones en las que se ha vulnerado la integridad de una persona, por acciones que atentan en contra de su dignidad o de la dignidad de sus familiares. Una ley, una política que no se vuelve operativa, que silencia al hombre o mujer que han padecido la violencia.

En el sinsabor de la inercia e inmanencia de la ley, de la política, aparece el sentimiento de los hombres y mujeres que quieren relatar al mundo, lo que les ha sucedido. Un relato que se representa en múltiples formas, entre ellas la pintura, la música, la literatura, etcétera. La imaginación del artista, se constituye en espacio para la libertad, para expresar lo que la violencia no deja pronunciar. Lo sublime se manifiesta como el sentimiento del espiritu, que permite la pronunciación de que no todo se terminó, sino que al contrario, lo sublime aparece como mínimo en el evidenciar el estar presente, el estar ahí. "Lo sublime es que del seno de esta inmanencia de la nada, sin embargo, suceda, tenga lugar algo que enuncie que no todo está terminado. Un simple aquí está, la ocurrencia más mínima en ese lugar" (Lyotard, 1998, p. 91).

Ante la experiencia de la violencia, que destruye la idea de lo bello de la vida, aparece la víctima como una receptora de la desilusión de lo bello, que en el impacto de la confrontación sólo puede dejarse llevar por lo que la ata, negándole toda posibilidad de realización de su libertad.

"La mente del sujeto queda congelada por un instante debido a una experiencia, en este caso la caída en el abismo, elemento básico de la producción de lo sublime. El abismo en su inmensidad, sobrepasa la capacidad de representación del individuo, quien está en presencia de un poder mayor que sí mismo" (Restrepo, 2004, p. 102).

Lo sublime en este marco de reflexión posee una característica importante, y es que ante la experiencia sublime que un expectante manifiesta en relación a una obra de arte que representa la fuerza de la violencia, se puede percibir, por un lado lo innombrable de lo acontecido, hay allí una especie de conocimiento, de idea que no es manifiesta, que está implícita en la obra de arte; y por otro, el sentimiento sublime ante la obra de arte por parte del observador, se experimenta por la idea que se descubre, una 
idea no manifiesta explícitamente, se manifiesta en la impresión que generan los matices de la obra, los trazos, los tonos, etc.

Lo sublime apela más bien a una ontología negativa. Lo que no impide que se espere de las artes este absurdo: que den testimonio en lo sensible de que algo falta a lo sensible o lo excede; su nombre no importa, es lo innombrable (Lyotard, 1996, p. 164).

La sensación ante la obra es la afectación que el sujeto experimenta de manera integral, en su mente y en su cuerpo, generando sentimientos que pueden modificar su concepción del mundo y de la vida. La experiencia ante lo sensible, es la afección ante algo que perturba la mente del sujeto afectado. El alma no se afecta por sí misma, sólo lo otro la afecta desde "afuera" (Lyotard, 1996, p.165).

La sensación dinamiza la vida de quien la experimenta, es una especie de fuerza que rompe con la inercia y permite la dinámica de una nueva percepción del mundo y de la existencia, la vida se hace sensible en la medida que se presentan nuevas formas de hacer la existencia, de generar mejores condiciones de vida para el desarrollo de la libertad de las personas.

Al respecto, Lyotard (1996) afirma:

La sensación provoca una fractura en una inexistencia inerte. La alerta, habría que decir, la existe. De allí que el ánima sólo existe cuando es forzada por lo que está afuera. Sólo basta algo para que la despierte, para que la haga existente, un color, un sonido, un sabor, algo que despierte el gusto, la vida, de quien se deja afectar por lo exterior.

Lo que centra nuestra atención en el marco de este escrito, es evidentemente, la necesidad de evidenciar la sensibilidad que se puede despertar ante una obra de arte que en su contenido, puede evidenciar hechos u acontecimientos violentos. Nos interesa reconocer que la afección que puede generar una de estas obras sobre la mente de un observador, es tal que puede transformar su imaginario, creando la posibilidad de que tal afectación remita a una nueva perspectiva de transformación de la persona, en su condición de vida particular.

Lo sublime en esta perspectiva, se presenta como la expresión subjetiva del artista o del observador de la obra, como la posibilidad de crear un medio ambiente nuevo en su vida, en el mundo en el que vive. Es el alma que despierta ante la experiencia sublime que produce la obra de arte, generando una ruptura con el continum de la inercia en la que está la vida social.

De allí que la nueva sensibilidad, permita el desarrollo de nuevas formas estéticas de la sociedad, en la que las sensibilidades subjetivas, puedan permear la sociedad y puedan así transformarla y crearla de acuerdo al interés, que desarrolla el bienestar de todos. Así el arte tendería a formar la realidad de la sociedad en su nuevo medio ambiente, en el que las presiones y las represiones sean superadas por la continua manifestación de lo sensible de sus miembros.

\section{Violencia en las obras de arte}

Para finalizar esta reflexión realizaré un acercamiento a obras de arte colombianas inscritas en el marco del concepto de violencia, resaltando la importancia que estas tienen en el marco de la construcción de un nuevo ambiente social de cultura de noviolencia.

La situación en la que vive la actual sociedad colombiana, en cuanto a violencia se refiere, es producto del constructo de una sociedad originada en la constante vulneración de los derechos humanos y de la denigración de la dignidad humana. Es una confrontación que lleva más de 40 años, en los que miles de personas han perecido producto de la violencia que se manifiesta de múltiples maneras, a saber, la de las guerrillas, la de los paramilitares, la del gobierno en concurso con grupos paramilitares y de los diferentes carteles de la droga, que se explicita en las masacres 
a poblaciones, campesinos, desplazamiento forzoso, desapariciones, secuestros, etcétera.

Muchas de estas formas de violencia perduran y continúan latentes en las comunidades donde se han radicado; la población frente a la constante presencia de estos grupos en muchos casos no han podido manifestar su dolor e inconformidad por los actos violentos, en muchas situaciones no han podido hacer efectiva la justicia, penalizando a quienes vulneraron sus derechos, o mataron a sus seres queridos.

Tanta violencia genera consecuencias que se ven reflejadas en otras formas de violencia que surgen del desplazamiento, de la pobreza, de la falta de recursos para sobrevivir, y en especial, cuando una familia llega a una ciudad desconocida a seguir luchando por su subsistencia, como ocurre en muchas localidades ${ }^{3}$ de la periferia de las grandes ciudades.

Otros casos de violencia que hacen parte de la historia de Colombia, se hacen visibles en monumentos que las víctimas han construido para no olvidar los acontecimientos y para representarse a sus seres queridos desaparecidos por la violencia. Como es el caso de Trujillo ${ }^{4}$ en el Valle.

La manera en que la población ha querido pronunciarse frente a la situación de violencia es el de sacar adelante un parque monumento como espacio de conmemoración de los actos violentos sufridos y de los que persisten en la actualidad. Un monumento en el cual se pueda representar a los seres queridos desaparecidos por la violencia en hechos aun no esclarecidos por la justicia o por el estado.
Lo anterior es muestra de la violencia en Colombia, en diferentes escenarios, pero que no son sino una mínima muestra de los hechos violentos que se han mantenido en la historia de este país. Lo que resulta pertinente en el marco de esta presentación, es evidenciar cómo los artistas en alguna forma mantienen una relación entre sus obras de arte y la violencia. Unos ejemplos que pueden ilustrar esta relación, donde la violencia es representada en una obra de arte se contienen, en las pinturas de Alejandro Obregón, como Violencia, óleo sobre lienzo ${ }^{5}$; Masacre del 10 de abril; Violento devorado por una fiera. De Alfonso Quijano tenemos La cosecha de los violentos y de Luis Ángel Rengifo Corte de Franela.

Los ejemplos anteriormente citados de obras que en su contenido permiten evidenciar hechos violentos, actos violentos o simplemente violencia, son una aproximación a la posibilidad de pensar en obras que permitan la exaltación del alma de quienes se acercan a observarlas. Violencia de Obregón, es una obra que a primera vista, permite reconocer que la muerte es la protagonista, el abandono del cuerpo de una mujer asesinada, abandonada en el horizonte, donde las tonalidades oscuras remiten a la tragedia del acontecimiento. El rostro de la muerte de la mujer, es sólo el espacio para estremecerse, es un rostro muy real, cuya palidez, entre los rastros de sangre y de tonalidades oscuras como de maltrato, surgen para decir lo que aconteció.

Los tonos oscuros de Violencia, remiten a un espacio en el que la penumbra es la que manifiesta o recuerda lo sombrío de la existencia, que se estremece ante la muerte, el abandono y la soledad misma de la muerte. A diferencia de

3 "Yo pienso que el cuento de la violencia en Ciudad Bolívar es en parte por la misma cultura de sobrevivencia, por el hambre, el desempleo, la falta de vivienda, gente que viene de muchos sectores de Colombia que son producto de otras violencias. Eso hace ver a Cuidad Bolívar como violenta en un cuarenta por ciento, y en el otro sesenta por ciento su imagen violenta es creada por los medios de comunicación" (Alape, 1995, p. 67).

4 Entre los años de 1984 y 1994, la población de Trujillo (departamento del Valle, República de Colombia) sufre por el conflicto entre diversos grupos la violencia, donde unas 342 personas han sido asesinadas en hechos que aún siguen impunes. Y es un pueblo que en cierta medida aún sigue enfrentándose a situaciones de violencia que no ha cesado y que posibilita el aparecimiento de nuevas víctimas. Además de esta situación es una población que lucha constantemente para que no sea olvidada, y en busca de la reconstrucción de su memoria y de la visibilización del conflicto que continua.

5 Ver reproducción de la obras en: http://www.banrepcultural.org/blaavirtual/revistas/credencial/junio1991/junio2.htm 
Violencia, masacre del 10 de abril, se presenta con unas tonalidades más claras, la muerte no aparece abandonada, el tumulto de cuerpos, hacen sentir la obra como más dinámica. Es una mezcla de colores, de opuestos, de rojo y azul, de blanco y negro, donde se resaltan los rostros de los asesinados, como si viviesen, pero no viven, son miradas cadavéricas.

La cosecha de los violentos de Alfonso Quijano, permite experimentar una sensación de desgarre ante la muerte, que parece recrearse en un árbol seco y con grandes ramas, que cubre la cantidad de cuerpos uniformemente ubicados bajo su estructura. Es como la contraposición entre el árbol de la vida -que es citado por la tradición judeo cristiana- y los frutos son la muerte.

La experiencia del espíritu que se puede tener ante estas obras, es aquella que despierta sensibilidades y que rompe con el sentir de la cotidianidad. La pregunta sobre el acontecimiento, se puede dejar de lado, aquí no interesa qué pasó ni cuándo aconteció. Lo que se manifiesta al espectador es el hecho mismo de la violencia. Y lo que dice la obra, habría de impactar a quienes la observan.

\section{Referencias bibliográficas}

Alape, A. (1995). La hoguera de las ilusiones. Bogotá: Planeta.

Amaya, A. M. (2009). La libertad entre lo visible y lo invisible. Revista de Estudios Sociales, 34.

Biblioteca Luis Ángel Arango del Banco de la República. Desde la muerte de Gaitán al Frente Nacional. Publicación digital en la página web de la Biblioteca Luis Ángel Arango del Banco de la República. http://www.lablaa.org/blaavirtual/revistas/credencial/junio1991/junio2.htm Consultada el 7 de junio de 2010.

Fry, K. (2004). The role of aesthetics in the politics of Jean-Francois Lyotard. Philosophy Today; 48(1), Research Library Core.
Esta ruptura que se presenta en la confrontación con el arte y la violencia, tendría que generar una nueva conciencia en las personas que las experimentan, permitiendo contemplar una nueva conciencia de la sociedad en la que viven. La capacidad persuasiva de la obra de arte habría de generar un cambio en las acciones de las personas.

En la nueva sensibilidad la capacidad persuasiva de la obra de arte es mayor, dada su capacidad de tocar la sensibilidades de las personas, las experiencias sublimes que se pueden experimentar permiten desarrollar una nueva dinámica de acción, como si la norma que es imperativa, dejase de ser imperativa y pasara a ser vivida porque el arte sí logra en cierta forma, persuadir por la impresión que deja en el alma de quienes se acercan a la obra de arte.

En esta medida, la obra de arte se puede convertir en una generadora de cultura de noviolencia, dada su capacidad de persuadir, de tocar las sensibilidades más profundas de la sociedad, posibilitando que la idea de una nueva sociedad se construya a partir de formas menos violentas y más sugestivas al desarrollo de la libertad de quienes viven en ella.

Lyotard, J. (1998). Lo inbumano. Buenos Aires: Manantial.

Madrid: Tecnos.

Marrero, M. (2008). La estética de la libertad y su expresión en Cintio Vitier. La Habana: Editorial Universitaria.

Marcuse, H. (1969). Un ensayo sobre la liberación. México: Editorial Joaquín Mortiz.

Restrepo, P. (2004). Lo sublime y el caos urbano: visiones apocalípticas de Medellín en la Virgen de los Sicarios de Fernando Vallejo. Chasqui, 33(1) 96-105. 